\title{
Experimental Evidence for a Light and Broad Scalar Resonance in $D^{+} \rightarrow \pi^{-} \pi^{+} \pi^{+}$Decay
}

\author{
Jussara M. de Miranda \\ for the \\ Fermilab E791 Collaboration \\ Centro Brasileiro de Pesquisas Físicas, Rio de Janeiro \\ E-mail: jussara@lafex.cbpf.br
}

Received 7 January, 2000

\begin{abstract}
From a sample of $1240 \pm 51 D^{+} \rightarrow \pi^{-} \pi^{+} \pi^{+}$we find $\Gamma\left(D^{+} \rightarrow \pi^{-} \pi^{+} \pi^{+}\right) / \Gamma\left(D^{+} \rightarrow K^{-} \pi^{+} \pi^{+}\right)=$ $0.0329 \pm 0.0015_{-0.0026}^{+0.0016}$. Using a coherent amplitude analysis to fit the Dalitz plot of this decay, we find strong evidence that a scalar resonance of mass $483_{-25}^{+26} \pm 17 \mathrm{MeV} / c^{2}$ and width $338_{-42}^{+45} \pm 21$ $\mathrm{MeV} / c^{2}$ produces a decay fraction of approximately one half.
\end{abstract}

\section{Introduction}

The lightest scalar and isoscalar resonance, predicted in models for spontaneous breaking with chiral symmetry such as the Nambu-Jona-Lazino linear $\sigma$ model [1] and its QCD extension[2], remain without an unambiguous experimental observation to this day. Experiments have presented controversial evidence for low-mass $\pi \pi$ resonances in partial wave analyses $[3,4,5]$. Determining the existence and characteristics of such particles have important consequences for the quark model [6], for understanding low energy $\pi \pi$ interactions[7, 8] and for understanding the $\Delta I=1 / 2$ rule[9].

Three-body decays of mesons containing heavy quarks often proceed as quasi-two body decays with resonant intermediate states. The large coupling to scalar resonances is the other frequent feature that makes charm decays a relatively clean laboratory for the study of scalar resonances. The large decay fractions of $D_{s} \rightarrow f_{0}(980) \pi$, and $D_{s} \rightarrow f_{0}(1370) \pi$ are good examples, together they account for almost $90 \%$ of the total $D_{s} \rightarrow \pi^{-} \pi^{+} \pi^{+}$width [10].

We present the study of the single Cabibbo suppressed decay $D^{+} \rightarrow \pi^{-} \pi^{+} \pi^{+}$. The amplitude analysis of the decay determine its resonance structure. We measure decay fractions to the established resonances[11]: $\rho^{0}(770), f_{0}(980), f_{2}(1270), f_{0}(1370)$, and $\rho^{0}(1450)$. We find that including an amplitude for a light broad scalar resonance improves our fit substantially. We measure the mass and width of such resonance to be $483_{-25}^{+26} \pm 17 \mathrm{MeV} / c^{2}$ and $338_{-42}^{+45} \pm 21$ $\mathrm{MeV} / c^{2}$. Referring to this $\pi^{+} \pi^{-}$resonance as

the $\sigma(500)$, we find that $D^{+} \rightarrow \sigma(500) \pi^{+}$accounts for almost half of the total decay rate.

\section{The data sample}

The data were produced by $500 \mathrm{GeV} / c \pi^{-}$interactions in five thin foils (one platinum, four diamond) separated by gaps of 1.34 to $1.39 \mathrm{~cm}$. The detector, the data set, the reconstruction, and the resulting vertex resolutions have been described previously [12]. After reconstruction, events with evidence of well-separated production (primary) and decay (secondary) vertices were retained for further analysis. From the 3 -prong secondary vertex candidates, we select a $\pi^{-} \pi^{+} \pi^{+}$sample with invariant mass ranging from 1.7 to $2.1 \mathrm{GeV} / \mathrm{c}^{2}$. All charged particles are assumed to be pions.

We require a candidate's secondary vertex position to be cleanly separated from the event's primary vertex position and from the closest target material. The sum of the momentum vectors of the three tracks from this secondary vertex must point to the primary vertex. The candidate's daughter tracks must pass closer to the secondary vertex than to the primary vertex, and must not point back to the primary vertex. The resulting invariant mass spectrum is shown in Fig. 1.

We fit the spectrum of Fig. 1 as the sum of $D^{+}$and $D_{s}^{+}$signals plus background. To account for the signals' non-Gaussian tails, we model each signal as the sum of two Gaussian distributions with the same centroid. We model the background as the sum of four components: a general combinatorial background, the reflection of the $D^{+} \rightarrow K^{-} \pi^{+} \pi^{+}$decay, reflections of $D^{0} \rightarrow K^{-} \pi^{+}$ plus one extra track, and $D_{s}^{+} \rightarrow \eta^{\prime} \pi^{+}$followed by 
$\eta^{\prime} \rightarrow \rho^{0}(770) \gamma, \rho^{0}(770) \rightarrow \pi^{+} \pi^{-}$. The $D^{+} \rightarrow K^{-} \pi^{+} \pi^{+}$ reflection is located below $1.82 \mathrm{GeV} / c^{2}$ in the $\pi^{-} \pi^{+} \pi^{+}$ spectrum. The other charm backgrounds populate the whole $\pi^{-} \pi^{+} \pi^{+}$spectrum. We use MC simulations to determine the shape of each identified charm background in the $\pi^{-} \pi^{+} \pi^{+}$spectrum. We assume that the combinatorial background falls exponentially with mass. The level of $D^{0} \rightarrow K \pi$ and $D_{s}^{+} \rightarrow \eta^{\prime} \pi^{+}$ backgrounds are determined using charm signal rates measured in our total event sample and branching ratios taken from the compilation by the Particle Data Group[11]. The parameters describing the combinatorial background and the level of the $K^{-} \pi^{+} \pi^{+}$reflection are determined from fitting the $\pi^{-} \pi^{+} \pi^{+}$distribution. The mass (centroid) and both Gaussian widths for each signal float in our fit. The fit finds $1240 \pm 51 \mathrm{D}^{+}$events and $858 \pm 49 D_{s}^{+}$events.

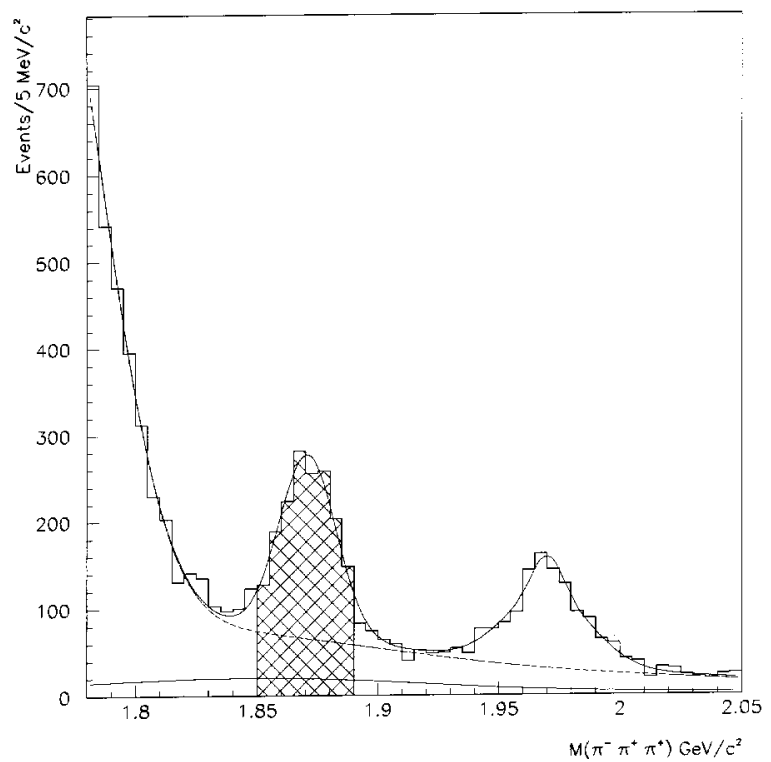

Figure 1. The $\pi^{-} \pi^{+} \pi^{+}$effective mass spectrum. Events in the hatched area are used for the Dalitz plot analysis.

To study the resonant structure of the decay $D^{+} \rightarrow$ $\pi^{-} \pi^{+} \pi^{+}$, we consider the 1686 candidates with invariant mass between 1.85 and $1.89 \mathrm{GeV}$. The integrated signal-to-background ratio in this range is about 2:1. Fig. 2 shows the Dalitz plot for these events. The horizontal and vertical axes are the squares of the $\pi^{+} \pi^{-}$invariant masses, and the plot has been symmetrized with respect to the two $\pi^{+}$'s. We see the clear bands corresponding to the $\rho^{0}(770) \pi^{+}$, with a valley in the central region, signature of the decay of the $D$ into a vector resonance and a $\pi$. Also clear is the band atributed to the $f_{0}(980) \pi^{+}$channel. We observe as well a large concentration of events in the low mass region. See Fig. 3 for simulations of the individual resonance contributions to Dalitz-plot.
We assume that the combinatorial background produces no particular structure in the Dalitz plot, apart from the shape imposed by the detector acceptance. This source is responsible for $72 \pm 9 \%$ of the overall Dalitz-plot backgound On the other hand, the charm backgound does produce specific structures. The shape, size and location of the charm backgound were obtained using Monte Carlo simulations and previously determined $D^{0}$ and $D_{s}$ production rates relative do $D^{+}$in our data sample. The $D^{0} \rightarrow K^{-} \pi^{+}$, represents only $4 \pm 1 \%$ of the background and is located in the symmetric corners of the Dalitz Plot. We associate the remaining $24 \pm 9 \%$ background to the $D_{s}^{+} \rightarrow \eta^{\prime} \pi^{+}$, $\eta^{\prime} \rightarrow \rho^{0}(770) \gamma, \rho^{0}(770) \rightarrow \pi^{+} \pi^{-}$that falls, obviuously, in the $\rho$ region, it is just distorted due to the kinematical cut on the $3 \pi$ invariant mass. This background differs from the signal decay to $\rho \pi$ in the sense that it does not present the valey in the central region, result of angular momentum conservation.

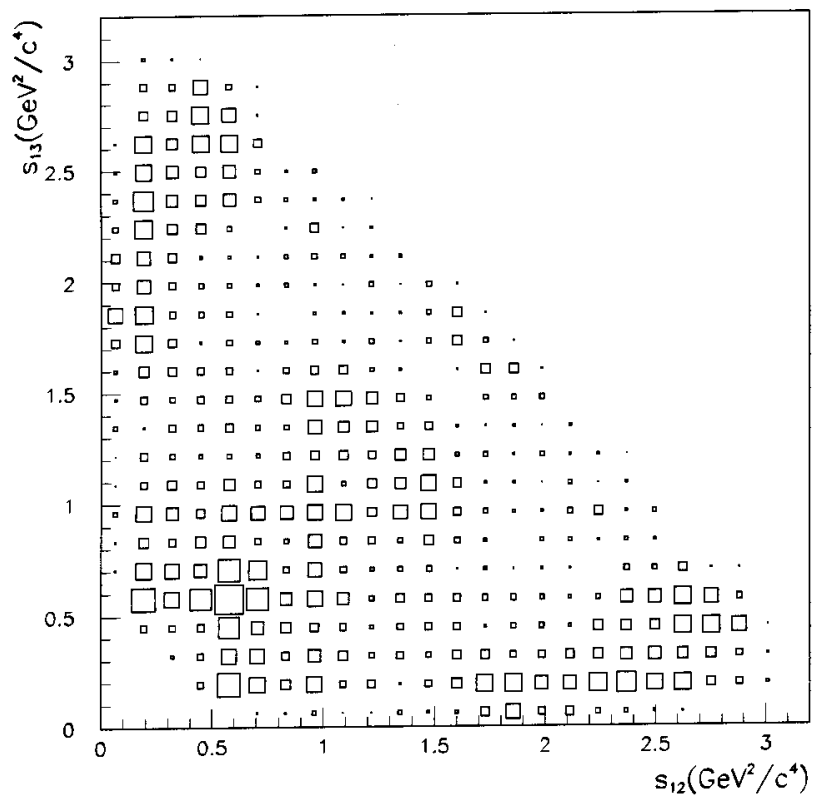

Figure 2. $D^{+} \rightarrow \pi^{-} \pi^{+} \pi^{+}$Dalitz plot distribution. Since we have two identical particles this distribution was symmetrized.

\section{The amplitude formalism}

The decay of a scalar hadron into 3 spinless daughter particles is completely specified with two degrees of freedom. The convenient choice of observable are the squared invariant mass of two two-body combination among the daughter particles. This choice is motivated by observing that the decay $D \rightarrow \mathbf{1} \mathbf{2} \mathbf{3}$ is written in terms of the invariant amplitude $\mathcal{A}$ as: 


$$
d \Gamma=\frac{1}{(2 \pi)^{3}} \frac{1}{32 M^{3}}\left\lceil\left.\mathcal{A}\right|^{2} d m_{\mathbf{1 2}}^{2} d m_{\mathbf{1 3}}^{2}\right.
$$

$M$ is the three-body invariant mass and $m_{i j}^{2}$ is the squared invariant mass of daughters $i$ and $j$.

The invariant amplitude $\mathcal{A}$ holds all the dynamics of the decay. The $m_{\mathbf{1 2}}^{2} \times m_{\mathbf{1 3}}^{2}$ scatter plot, the DalitzPlot, is a display of this dynamics. The next step is to compose a model for $\mathcal{A}$ and investigate how it compares to the data.

The decay through resonant intermediate states, $j$, are viewed as s-channel processes where the resonance plays the role of massive propagators, represented by relativistic Breit-Wigner functions, $B W_{j}{ }^{1}$. Momentum dependent form factors, $F_{D}$ and $F_{R}$ describe the non-pointlike nature of the $D$ meson and the resonance respectively. The angular momentum conservation is taken care by the function $\mathcal{M}_{j}^{J}$. Each resonant amplitude, $\mathcal{A}_{j}$ is writen as:

$$
\begin{aligned}
\mathcal{A}_{j} & =B W_{j} \times F_{D} \times F_{R} \times \mathcal{M}_{j}^{J} \\
B W_{j} & =\frac{1}{m_{\pi \pi}^{2}-m_{0}^{2}+i m_{0} \Gamma_{j}\left(m_{\pi \pi}\right)}
\end{aligned}
$$

with

$$
\Gamma\left(m_{\pi \pi}\right)=\Gamma_{0} \frac{m_{0}}{m_{\pi \pi}}\left(\frac{p^{*}}{p_{0}^{*}}\right)^{2 J+1} \frac{F_{R}^{2}\left(p^{*}\right)}{F_{R}^{2}\left(p_{0}^{*}\right)} .
$$

Above $m_{\pi \pi}$ is the invariant mass of the two pions forming a spin-J resonance. The functions $F_{R}$ are the Blatt-Weisskopf damping factors [14] that depend on the spin of the resonance: $F_{0}=1$ for spin 0 particles, $F_{1}=1 / \sqrt{1+\left(r p^{*}\right)^{2}}$ for spin 1 and $F_{2}=$ $1 / \sqrt{9+3\left(r p^{*}\right)^{2}+\left(r p^{*}\right)^{4}}$ for spin 2. The parameter $r$ is the radius of the resonance ( $\sim 3 \mathrm{fm})$ [14] and $p^{*}=p^{*}(m)$ the momentum of decay particles at mass $m$, measured in the resonance rest frame. The spin part of the amplitude $\mathcal{M}_{j}$ is defined equal to 1 for a spin-0 resonance, $-2\left|\mathbf{p}_{\mathbf{3}} \| \mathbf{p}_{\mathbf{1}}\right| \cos \theta$ for spin1 and $\frac{4}{3}\left(\left|\mathbf{p}_{\mathbf{3}} \| \mathbf{p}_{\mathbf{1}}\right|\right)^{2}\left(3 \cos ^{2} \theta-1\right)$, where $\mathbf{p}_{\mathbf{3}}$ is the 3 -momentum of the bachelor pion and $\mathbf{p}_{\mathbf{1}}$ is the 3 momentum of the other like charge pion, both measured in the resonance rest frame; and $\theta$ is the angle between pions 1 and 3. Finally, each resonant amplitude is Bose symmeterized $\mathcal{A}_{i}=\mathcal{A}_{i}[(\mathbf{1 2}) \mathbf{3}]+\mathcal{A}_{i}[(\mathbf{1 3}) \mathbf{2}]$

The non-resonant amplitude is represented by a constant. The overall amplitude is written as a coherent sum of all the contributions:

$$
\mathcal{A}=c_{0} \mathcal{A}_{0}+\sum_{j=1}^{n} c_{j} \mathcal{A}_{j},
$$

where $c_{j}=a_{j} e^{i \delta_{j}}$ are complex coefficients, they represent the relative contributions of each decay mode and as well as final state hadronic interactions. These coefficients are the unknowns that should be extracted from fits to the data. Fig. 3 we show the Dalitz-plot of each individual resonant state that could contribute for the above sum in the $D^{+} \rightarrow \pi^{-} \pi^{+} \pi^{+}$decay. Notice that being coefficients and the individual amplitudes complex quantities, interference effects will take place when all the pieces act together.
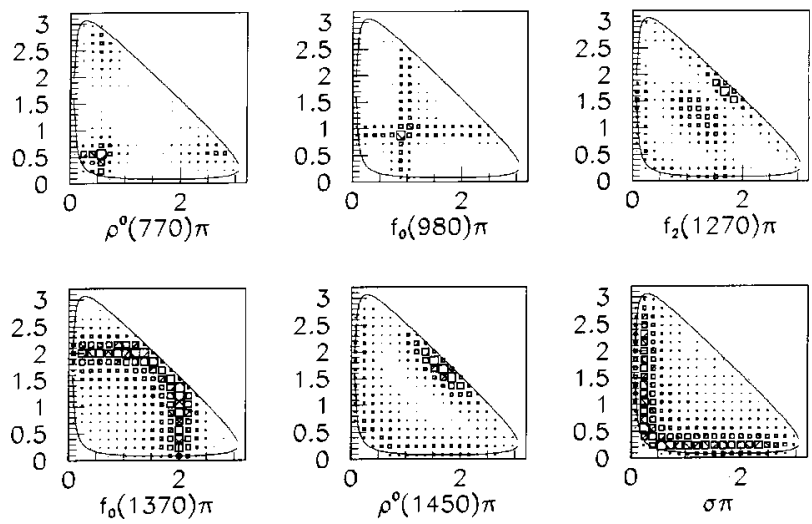

Figure 3. Dalitz-plot of the individual resonant contributions to the $D^{+} \rightarrow \pi^{-} \pi^{+} \pi^{+}$decay.

To obtain the parameters $a_{j}$ and $\delta_{j}$ we do a maximum likelihood $\mathcal{L}$ fit to the data using MINUIT [15]. In practice the program finds parameters that minimize the quantity $w=-2 \ln (\mathcal{L})$. We compute the likelihood, $\mathcal{L}$, in terms of signal, $\mathcal{P}_{S}$, and background, $\mathcal{P}_{B}$, probability functions (PDF's) of the $\pi^{-} \pi^{+} \pi^{+}$invariant mass, $M$, and the Lorentz invariants $s_{12} \equiv m_{12}^{2}$ and $s_{13} \equiv m_{13}^{2}$ :

$$
\mathcal{L}=\prod_{j=1}^{1686}\left[\mathcal{P}_{S}+\mathcal{P}_{B}\right]_{j},
$$

${ }^{1}$ For the $f_{0}(980) \pi^{+}$we use a coupled channel Breit-Wigner, following the parameterization of the WA76 Collaboration [13],

$$
B W_{f_{0}(980)}=\frac{1}{m_{\pi \pi}^{2}-m_{0}^{2}+i m_{0}\left(\Gamma_{\pi}+\Gamma_{K}\right)},
$$

with

$$
\Gamma_{\pi}=g_{\pi} \sqrt{m_{\pi \pi}^{2} / 4-m_{\pi}^{2}},
$$

$$
\Gamma_{K}=\frac{g_{K}}{2}\left(\sqrt{m_{\pi \pi}^{2} / 4-m_{K^{+}}^{2}}+\sqrt{m_{\pi \pi}^{2} / 4-m_{K^{0}}^{2}}\right)
$$




$$
\mathcal{P}_{S}=\frac{1}{N_{S}} g(M) \varepsilon\left(s_{12}, s_{13}\right)|\mathcal{A}|^{2} .
$$

In this equation $N_{S}$ is the normalization constant, $\varepsilon\left(s_{1}, s_{2}\right)$ is the acceptance factor, $g(M)$ is a gaussian function describing the signal's $\pi^{-} \pi^{+} \pi^{+}$observed invariant mass spectrum. $\mathcal{A}$ is the general amplitude given by equation 5 , function of the fit parameters $a_{j}$ and $\delta_{j}$. With the exception of the $\sigma$, the masses and widths of all the resonances were fixed for all fits reported here. In table 1 we list mass and width values used.

Table 1. Masses and widths of the resonances used in the Dalitz plot fit. Values for $f_{0}(980)$ and $f_{0}(1370)$ came from the Dalitz-plot analysis of the $D_{s} \rightarrow$ $\pi^{-} \pi^{+} \pi^{+}$signal shown in Fig. 1, all the others are listed [11].

\begin{tabular}{llll}
\hline resonance & mass $(\mathrm{GeV})$ & width & source \\
\hline$f_{0}(980)$ & 0.978 & $\begin{array}{l}g_{\pi}=0.083 \\
g_{K}=-0.03\end{array}$ & fit \\
& & 0.150 & PDG \\
$\rho^{0}(770)$ & 0.769 & 0.185 & PDG \\
$f_{2}(1270)$ & 1.275 & 0.170 & fit \\
$f_{0}(1370)$ & 1.438 & 0.310 & PDG \\
$\rho^{0}(1450)$ & 1.465 & & \\
\hline
\end{tabular}

The background distribution is given by

$$
\mathcal{P}_{B}=b(M) \sum_{i=1}^{3} \frac{b_{i}}{N_{B_{i}}} \mathcal{B}_{i}\left(s_{12}, s_{13}\right),
$$

$b(M)$ is the function describing the background distribution in the $\pi^{-} \pi^{+} \pi^{+}$spectrum, $b_{i}$ are the relative amount of each background type and $N_{B_{i}}$ are the corresponding normalization constants. $\mathcal{B}_{i}$ are the probability distributions in the Dalitz-plot of each background type, they are displayed in Fig. 4. There are no free parameters in the background description although we vary $b_{i}$ to estimate the systematic uncertainty in the measured parameters. Fig. $4 \mathrm{a}$ is a plot of $\mathcal{P}_{B}$ and in $4 \mathrm{~b}$ we plot the detector acceptance.
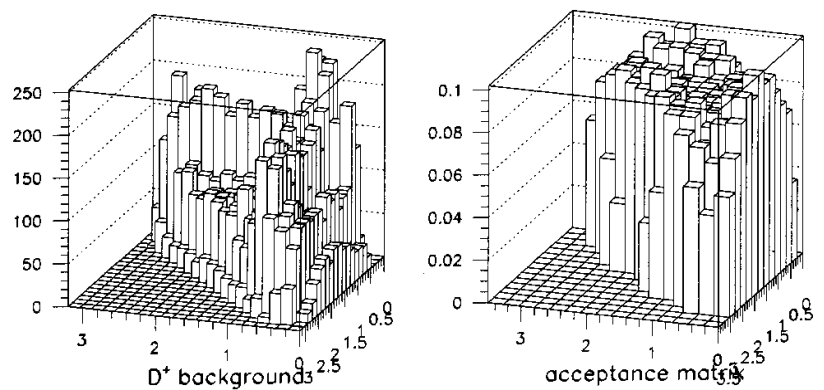

Figure 4. a) Background distribution b) Detector acceptance.

\section{Results}

We have tried many different models for the signal amplitude, particularly in its respect to the $\sigma(500)$ resonance. In our first model, which we will refer to as Fit 1 the signal PDF includes a non-resonant amplitude and amplitudes for $D^{+}$decaying to a $\pi^{+}$and any of the five well-stablished resonances [11]: $\rho^{0}(770), f_{0}(980)$, $f_{2}(1270), f_{0}(1370)$, and $\rho^{0}(1450)$. The fit extracts magnitudes and phases of each of the amplitudes along with the error matrix for those parameters. We calculate the decay fractions for each amplitude as:

$$
f_{j} \equiv \frac{\int d s_{12} d s_{13}\left|c_{j} \mathcal{A}_{j}\right|^{2}}{\int d s_{12} d s_{13} \sum_{j k}\left|c_{j} \mathcal{A}_{j} c_{k}^{*} \mathcal{A}_{k}^{*}\right|^{2}}
$$

The integration runs over the entire Dalitz-Plot. Notice that due to interference effects the fractions of all modes do not necessarily adds up to one. For this reason we are not able to measure exactly the intermediate state branching ratios. The Fit 1 results are collected in table 1 . This fit found the minimim value $w=-2 \ln (\mathcal{L})=-5138$. In this model, the nonresonant and the $\rho^{0}(1450) \pi^{+}$apear to dominate, followed by the $\rho^{0}(770) \pi^{+}$amplitude. The qualitative features of this fit are similar to those reported by E691 [16] and E687 [17].

Table 2. Fit 1 for $D^{+} \rightarrow \pi^{+} \pi^{-} \pi^{+}$decay

\begin{tabular}{lll} 
mode & Magnitude & Fraction $(\%)$ \\
\hline$\rho^{0}(770) \pi^{+}$ & Phase & \\
& $\begin{array}{l}1 \text { (fixed) } \\
\text { (fixed) }\end{array}$ & $20.2 \pm 2.7$ \\
non-res. & $1.25 \pm 0.23$ & $31.6 \pm 9.0$ \\
& $(141.0 \pm 9.7)^{\circ}$ & \\
$f_{0}(980) \pi^{+}$ & $\begin{array}{l}0.64 \pm 0.08 \\
\end{array}$ & $8.1 \pm 1.4$ \\
$f_{2}(1270) \pi^{+}$ & $0.63 \pm 0.08$ & $8.0 \pm 2.1$ \\
& $(85.9 \pm 16.6)^{\circ}$ & \\
$f_{0}(1429) \pi^{+}$ & $0.75 \pm 0.11$ & $11.5 \pm 2.9$ \\
& $(142.7 \pm 9.7)^{\circ}$ & \\
$\rho^{0}(1450) \pi^{+}$ & $1.15 \pm 0.12$ & $26.8 \pm 3.8$ \\
& $(28.6 \pm 13.8)^{\circ}$ & \\
\hline
\end{tabular}

To acess the quality of each fit we developed a fast Monte Carlo (MC) program which produces DalitzPlot event densities accounting for signal and backgound PDF's, including detector efficiency and resolution. Comparing the MC density distribution generated using magnitudes and phases of the amplitudes 
given in Fit 1 with that for the data, we produde a $\chi^{2}$ distribution shown in Fig. 5. We observe bad $\chi^{2}$ bins spread thoughtout the Dalitz-Plot with particularly bad bins in the low $\pi \pi$ mass region. The $\chi^{2}$ summed over all bins is 243.0 for 162 degrees of freedom, giving $\chi^{2} / D O F=1.50$. F Fig. $5 \mathrm{~b}$ displays the $\pi^{+} \pi^{-}$square mass projection for data and fast MC. While the peaks corresponding to the $\rho^{0}(770) \pi^{+}$and the $f_{0}(980) \pi^{+}$are well represented by the model, there is a discrepancy at the lower mass suggesting, along with the relatively poor $\chi^{2}$, the possibility of another resonant amplitude.
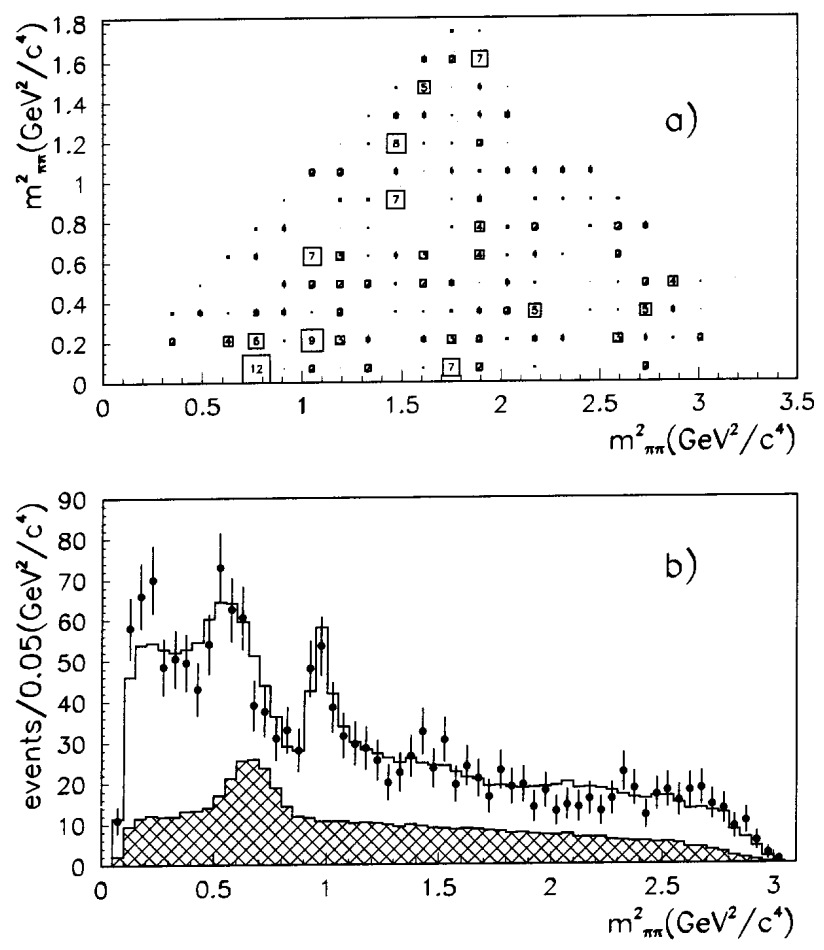

Figure 5. Fit 1: a) $\chi^{2}$ distribution b) $s_{12}$ and $s_{13}$ projections for data (error bars), fast MC (solid) and the shaded area is the backgouhd distribution.

To investigate the possibility that another resonance contributes to the $D^{+} \rightarrow \pi^{-} \pi^{+} \pi^{+}$decay, we add a seventh amplitude to the signal PDF. We allow the mass and the width to float and asusume a scalar angular distribution. This fit (Fit 2) finds values of $483_{-25}^{+26}$ $\mathrm{MeV} / \mathrm{c}^{2}$ and $338_{-42}^{+45} \mathrm{MeV} / \mathrm{c}^{2}$ respectively for mass and width of this sixth resonant state, that we are refering to as $\sigma(500)$. The other parametes of the fit are listed in table 3 . We obtained for Fit $2, w=-2 \ln (\mathcal{L})=-5248$

In Fit 2, the $\sigma(500)$ amplitude produces the largest decay fraction , $44 \%$, with a relatively small statistical error, $9 \%$. The non-resonant fraction, which at $(32 \pm 10) \%$ was the largest in Fit 1 , is now only $(10 \pm 7) \%$. The first reported errors are statistical followed by the systematic. The possible sources of systematic uncertainties are the background model, the detector acceptance and the $\sigma(500)$ mass and width. We also investigate the impact of having pointlike hadrons, neglecting the form factors in the model.

Fig. 6a shows the distribution of the $\chi^{2}$ of the data if compared with a fast MC sample generated with the parameters out of Fit 2. When we project this model onto the Dalitz plot, $\chi^{2} / D O F$ becomes 149/162. Along with the significative improvement in the global $\chi^{2}$ we note also the lack of prefered bad spots in the $\chi^{2}$ distribution. In the $\pi^{+} \pi^{-}$squared mass projections, Fig. $6 \mathrm{~b}$, we see clearly that this model describes the data well, including the the low mass region with the peak at $0.2 \mathrm{GeV}^{2} / c^{4}$.
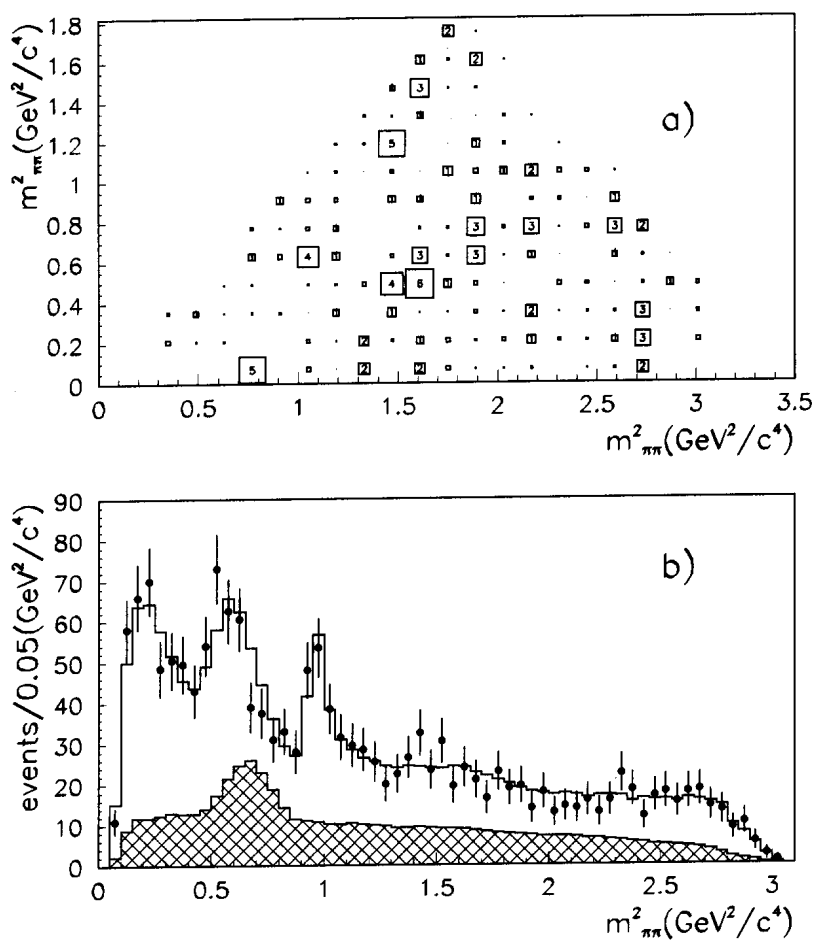

Figure 6. Fit 2: a) $\chi^{2}$ distribution b) $s_{12}$ and $s_{13}$ projections for data (error bars), fast MC (solid) and the shaded area is the backgouhd distribution.

We used hundreds of fast MC samples to test the accuracy of the errors estimated by the fitter. The total number of signal and backgound events of these MC ensembles varied according to gaussian distributios centered in the values relative to our data set. The central value of al parameters are reproduced accurately and the width of the $\sigma(500)$ fraction distribution is 0.12 . To compare the ability of the two models of representing the data we used the quantity $\Delta w=-2\left(\mathcal{L}_{\sigma}-\mathcal{L}_{0}\right)$, where $\mathcal{L}_{\sigma}$ is calculated, for any given event sample, using the parameters listed in table 3 , similarly $\mathcal{L}_{0}$ are 
calculated using the parameters of table 2 . In the data $\Delta w=110$. In Fig. 7a the hashed area is the distribution of $\Delta w$ for samples of fast MC generated with the parameters of Fit 2 (table 3 ). The solid line on the same figure was obtained using fast MC samples generated with the parametes of Fit 1 . The triangle marks the data point. From this execise we conclude that the data event distribution is compatible with the model that includes the $\sigma(500)$ and is not statisticaly compatible with the model that includes only well-stablished resonat modes.

Table 3. Fit 2 for $D^{+} \rightarrow \pi^{+} \pi^{-} \pi^{+}$decay.

\begin{tabular}{lll}
\hline \multirow{2}{*}{ mode } & $\begin{array}{l}\text { Magnitude } \\
\text { Phase }\end{array}$ & Fraction(\%) \\
\hline$\sigma \pi^{+}$ & $\begin{array}{l}1.21 \pm 0.22 \pm 0.06 \\
(205.7 \pm 8.0 \pm 5.2)^{\circ}\end{array}$ & $44.3 \pm 9.4 \pm 2.1$ \\
$\rho^{0}(770) \pi^{+}$ & $\begin{array}{l}1(\text { fixed }) \\
0(\text { fixed })\end{array}$ & $30.5 \pm 3.1 \pm 2.2$ \\
non-res. & $\begin{array}{l}0.57 \pm 0.19 \pm 0.09 \\
(74.5 \pm 17.8 \pm 5.7)^{\circ}\end{array}$ & $9.9 \pm 7.0 \pm 2.7$ \\
$f_{0}(980) \pi^{+}$ & $\begin{array}{l}0.46 \pm 0.05 \pm 0.02 \\
(163.3 \pm 10.3 \pm 3.4)^{\circ}\end{array}$ & $6.6 \pm 1.3 \pm 0.4$ \\
$f_{2}(1270) \pi^{+}$ & $\begin{array}{l}0.80 \pm 0.06 \pm 0.03 \\
(53.3 \pm 7.5 \pm 2.9)^{\circ}\end{array}$ & $18.9 \pm 2.5 \pm 0.4$ \\
$f_{0}(1429) \pi^{+}$ & $\begin{array}{l}0.30 \pm 0.09 \pm 0.03 \\
(107.1 \pm 17.8 \pm 0.6)^{\circ}\end{array}$ & $2.5 \pm 1.5 \pm 0.8$ \\
$\rho^{0}(1450) \pi^{+}$ & $0.19 \pm 0.09 \pm 0.02$ & $1.1 \pm 1.0 \pm 0.3$ \\
& $(339.2 \pm 27.5 \pm 10.9)^{\circ}$ & \\
\hline
\end{tabular}

It remains to be answered if the extra scalar resonance is the only possible explanation for the data distribution. With the intention of answering this question we tried some other models. We allowed the seventh amplitude to be a vector, $\sigma_{\text {vector }}$ or a tensor, $\sigma_{\text {tensor }}$ resonance and we tried as well a toy Breit-Wigner without a phase, $\sigma_{B W 2}$. The toy model tests the phase variation expected by a Breit-Wigner amplitude. In each case we allowed the mass and width of the new object to float. The vector resonance model converges to poorly defined values of the mass and width: $719 \pm 182$ $\mathrm{MeV} / c^{2}$ and $1156 \pm 879 \mathrm{MeV} / c^{2}$; the tensor model to more poorly defined values: $2223 \pm 607 \mathrm{MeV} / c^{2}$ and
$-1096 \pm 1269$; and the toy model to $431 \pm 12 \mathrm{MeV} / c^{2}$ and $269 \pm 35 \mathrm{MeV} / c^{2}$. As a test of the models, we again project the the vector, tensor, and toy models onto the Dalitz plot and obtain $\chi^{2} / D O F=194 / 162,155 / 162$, and $158 / 162$, respectively. For these models, we also find $\Delta w=78,16$, and 31 where MC experiments pre$\operatorname{dict}\langle\Delta w\rangle \approx 61,51$, and 27 when the data is generated with the scalar parameters and the negatives of those values when the MC data is generated according to the vector, tensor, and toy model parameters, as show Figs. $6 \mathrm{~b}, \mathrm{c}$ and $\mathrm{d}$. These statistical tests strongly exclude the vector model. They clearly prefer the scalar model to the tensor and toy models. Note that the central value for the tensor mass is well above threshold for $D^{+}$decay and the negative width is an indication that no physically meaningful tensor resonance fits the data. In the toy model, the extra amplitude interferes strongly with a large non resonant amplitude, leading to an unphysically large sum of resonant fractions.

\section{Conclusion}

In summary, from $1240 \pm 51 D^{+} \rightarrow \pi^{-} \pi^{+} \pi^{+}$we have measured $\Gamma\left(D^{+} \rightarrow \pi^{-} \pi^{+} \pi^{+}\right) / \Gamma\left(D^{+} \rightarrow K^{-} \pi^{+} \pi^{+}\right)$. In an amplitude analysis of a sample with $\mathrm{S}: \mathrm{B} \approx 2: 1$ we find strong evidence that a scalar resonance with mass $483_{-25}^{+26} \pm 17 \mathrm{MeV} / c^{2}$ and width $338_{-42}^{+45} \pm 21 \mathrm{MeV} / c^{2}$ produces a decay fraction $\approx 50 \%$. Alternative explanations of the data fail to describe it as well. The prominence of an isoscalar $+\pi^{+}$amplitude in this decay accords well with our observation that isoscalar $+\pi^{+}$amplitudes produce a large majority of all $D_{s}^{+} \rightarrow \pi^{-} \pi^{+} \pi^{+}$ decays[10].

\section{Acknowledgements}

We gratefully acknowledge the assistance of the staffs of Fermilab and of all the participating institutions. This research was supported by the Brazilian Conselho Nacional de Desenvolvimento Científico e Tecnológico, CONACyT (Mexico), the Israeli Academy of Sciences and Humanities, the U.S. Department of Energy, the U.S.-Israel Binational Science Foundation, and the U.S. National Science Foundation. Fermilab is operated by the Universities Research Association, Inc., under contract with the U.S. Department of Energy. 

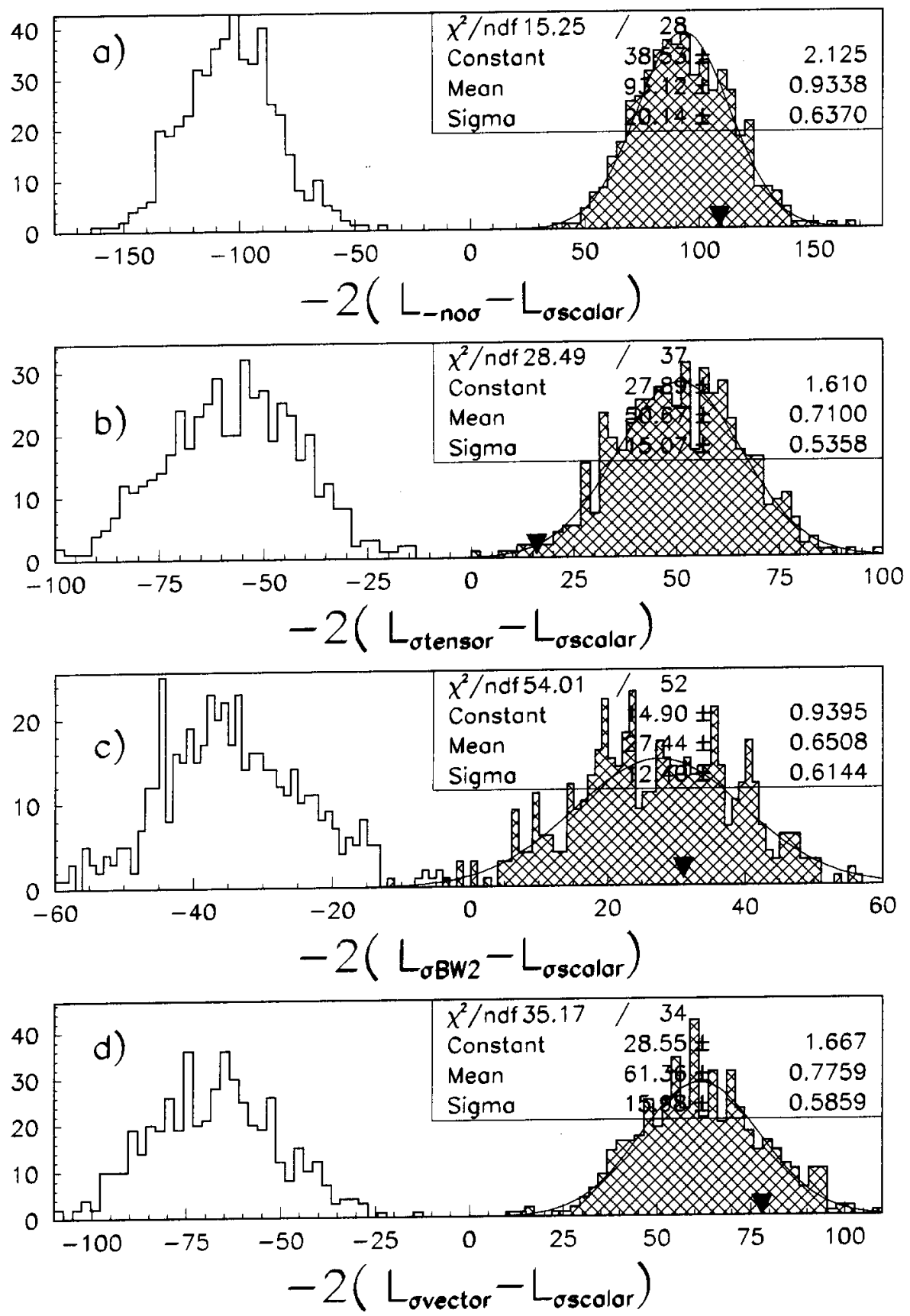

Figure 7. $\Delta w=-2\left(\mathcal{L}_{i}-\mathcal{L}_{\text {Gscalar }}\right)$. In all plots the hashed histograms were obtained from Fast MC samples generated according to the parameters of table 3. The triangles are the data points and for the solid line histograms we used fast MC generated by the model without a $\sigma$ resonance (a), with the $\sigma_{\text {tensor }}(\mathrm{b})$, with the toy Breit-Wigner without phase (c) and with the $\sigma_{\text {vector }}$.

\section{References}

[1] Y. Nambu and G.Jona-Lasinio, Phys. Rev. 122, 345 (1961)

[2] R. Delburgo, M.S. Scadron, Phys. Rev. Lett 48, 379 (1982).

[3] WA102 Collaboration, D. Barberies et al., Phys. Lett. B 453, 332 (1999).

[4] CLEO Collaboration, D.M. Asner et al., hep$\mathrm{ex} / 9902022$
[5] GAMS Collaboration, D. Alde et al., Phys. Lett. B 397, 350 (1997).

[6] N. Tornqvist, hep-ph/9904342. To appear in the Frascati Physics Series Vol. XXX (1997).

[7] K.Erkelene, Phys. Rep. C 13, 190 (1974).

[8] M.R. Pennington, hep-ph/9905241.

[9] T. Morozumi, C.S. Lim and A.I.Sanda, Phy. Rev. Lett. 65, 404 (1990). 
[10] E791 Collaboration, "Study of the $D_{s}^{+} \rightarrow \pi^{-} \pi^{+} \pi^{+}$decay and measurement of $f_{0}$ masses and widths". To appear at the proccedings of DAFNE99. http://wwwsis.lnf.infn.it/dafne99.

[11] Particle Data Group, C. Caso et al., Eur. Phys. Jour. C 3, 1 (1998).

[12] Eue. Phys. J. Direct c 4, 1 (1999) and references therein; J. A. Appel, Ann. Rev. Nucl. Part. Sci. 42, 367 (1992), D. J. Summers et al., Proceedings of the XXVII $^{\text {th }}$ Rencontre de Moriond, Electroweak Interactions and Unified Theories, Les Arcs, France (15 - 22 March 1992) 417; S. Amato et al., Nucl. Instr. Meth. A 324, 535 (1993).
[13] WA76 Collaboration, T.A. Armstrong et al., Z. Phys. C 51, 351 (1991).

[14] Argus Collaboration, Albrecht H. et al. Phys. Lett. B 308, 435 (1993).

[15] F. James, "MINUIT, Function Minimization and Error Analysis", CERN Program Library long Write-up D506 (1994).

[16] E691 Collaboration, Anjos, J. et al. Phy. Rev. Lett. 62, 125 (1989).

[17] E687 Collaboration, Frabetti P.L., et al. Phys. Lett. B 407, 79 (1997). 